\title{
Research on the Measuring Equipment Management Methodology for the manufacturing process
}

\author{
An Haixia ${ }^{1, a}$, Wang Meiqing ${ }^{2, b}$, Huang Ming ${ }^{2, c}$ \\ School of Mechanical Engineering and Automation, Beihang University, Beijing, 100191, China \\ aahxbuaa@126.com, bwangmq@buaa.edu.cn, cming_todobetter@163.com
}

Keywords: Measuring equipment management; Manufacturing process; Measurement system analysis (MSA); Periodical verification \& calibration planning

\begin{abstract}
The methodology of measuring equipment management for the manufacturing process, the use of measuring equipment, the health condition and the verification period planning for the measuring equipment is proposed on the basis of the research on the measuring equipment management and application in aviation manufacturing enterprises. In this paper the technology to support the process of collecting the data from verification \& calibration and manufacturing process is presented, which guarantees the data is real-time, true and accurate. The measurement system analysis (MSA) is introduced on the basis of the process and the data collected to decide the stability of the measuring equipment and provide proposal to the periodical verification \& calibration planning of measuring equipment.
\end{abstract}

\section{Introduction}

The measuring equipment management is crucial to the management of the whole manufacturing process no matter in manufacturing, medical or service industry. It is difficult for enterprises to provide satisfactory products for customers without the assist of the measuring equipment and the restriction of the measurement results. The computer technology has been integrated in managing measuring equipment with the development of the computer technology and informatization. Many information systems managing the measuring equipment effectively have been built, but they generally focused on the independent control of the standing information and periodical verification management but without tight connection with production process management because of the lake of understanding and attention of measuring equipment management ${ }^{[1],[2]}$, so it is difficult for the systems to guarantee the integrity, authenticity and accuracy of product data and to evaluate the health condition and influence of the measuring equipment which are used to collect the data.

Measuring equipment management is essential for manufacturing enterprise, which exits in the whole life cycle of the product, and it is an important part of the quality management, especially in the manufacturing process ${ }^{[3]}$. The control process also demands the preventive planning of equipment maintenance to ensure the accuracy, manufacturing capability and the continuous process. On the country the traditional periodical verification of measuring equipment management is to set a fix time as the period when the equipment is purchased, when the year/month period is over, the equipment is sent to verified/calibrated. But this is not suitable for the real production process because there are always factors impacting the measuring equipment accuracy exit, for example, operating frequency, maintenance status and stability of measuring equipment. Measurement system analysis (MSA) is feasible. It can proof that the main variation is from process but measurement system, and the measurement system is capable for the process, so that we can manage the measurement system analysis on the basis of the data and its statistical results. We conclude several problems in the traditional measuring equipment management model:

1) The capability to guarantee the data collected in the manufacturing process is true and reliable. The traditional measuring equipment management is independent from manufacturing process management, so the measuring data is collected from the machining control card which is written by inspectors. The pattern not only can't ensure the data's quality but also can't build the connection between the data and equipment to know impact of measurement to the quality 
problem.

2) The assurance of the measuring equipment of keeping healthy and meeting the use. The traditional management model is periodical verification to give simple status flag like qualified or unqualified without details, MSA cannot be handled by these status flag. But there are accidents in the process of use or transportation of the measuring equipment that may reduce accuracy, and lower the production quality and make loss of economy and time.

3) The possibility of measurement system analysis (MSA). Measuring equipment serve the production process, so their capacity and performance is very important. Without the restriction in the process of inspecting the equipment, these ill measuring equipment may be used, which would reduce the quality and the value of the manufacturing process.

\section{Methodology of Measuring Equipment Management for the process of manufacture}

The core of the measuring equipment management methodology is facing the manufacturing process and information collection and analysis for data is the soul of the quality management information system, only by collecting the true, reliable and real-time data can we analyze and statistic the data and provide practicable scheme to improve the quality and productive value. Measuring equipment are used to support the production process and guarantee the production quality, so the measuring equipment can be better used if it connected more with the production process.

Foundation of measuring equipment management. To support the manufacturing process more effectively, the strategy is imposed in measuring equipment management. From the perspective of manufacturing process, the measuring equipment are used to collect record and retrospect the parts feature information in the whole process. From the perspective of measuring equipment management itself, lots information is produced, including operating counts, operating frequency and maintenance counts, the record of the verification and calibration is especially important. The traditional measuring equipment management only record and update the status of the verification and maintenance, using qualified or unqualified to judge the verification and using repair or scrap as the result, but it is impossible to know the stability or historical verification or current status by MSA with these simple information.

Fig. 1 shows the ER diagram of the feature and verification \& calibration information collecting part of the measuring equipment management. In the figure the process collected the information from the whole business flow on the basis of measuring equipment. The information code, specialty, range and precision of the equipment are the basic of the machining and verification process. In the verification process, the information of Verification Room, operators and verification records are collected gradually, the measuring value and related data included. While in the production process the product, process and feature information and related data is received.

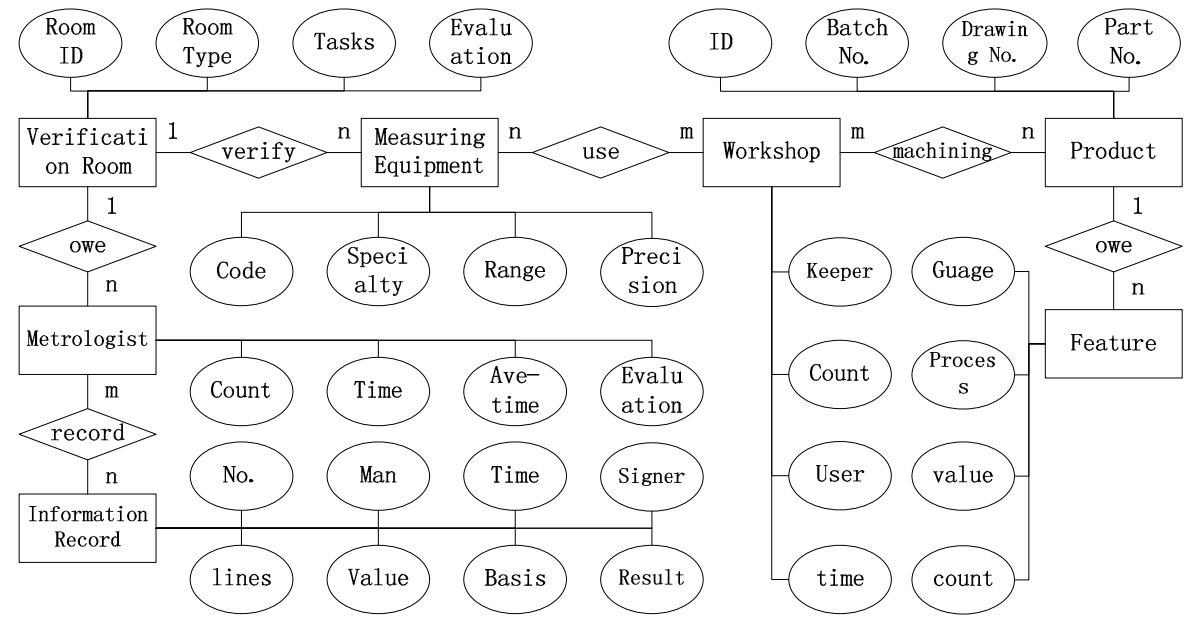

Fig. 1 ER diagram of measuring equipment information collecting

Quality assurance of Production field data collection. Manufacturing process information 
involves the data accepted directly or indirectly, including parameter of machining process, workload of operator and environment parameter. The Optimal Control for plant-wide Production Processes is proposed because of product value, quality and quantity exits the whole production process, and measurements which are the tools and basic of information collection is essential to get high-quality information for the manufacturing process ${ }^{[4]}$.In this paper, the information collected in manufacturing process is machining feature, measured value received by operators or the profession inspection department.

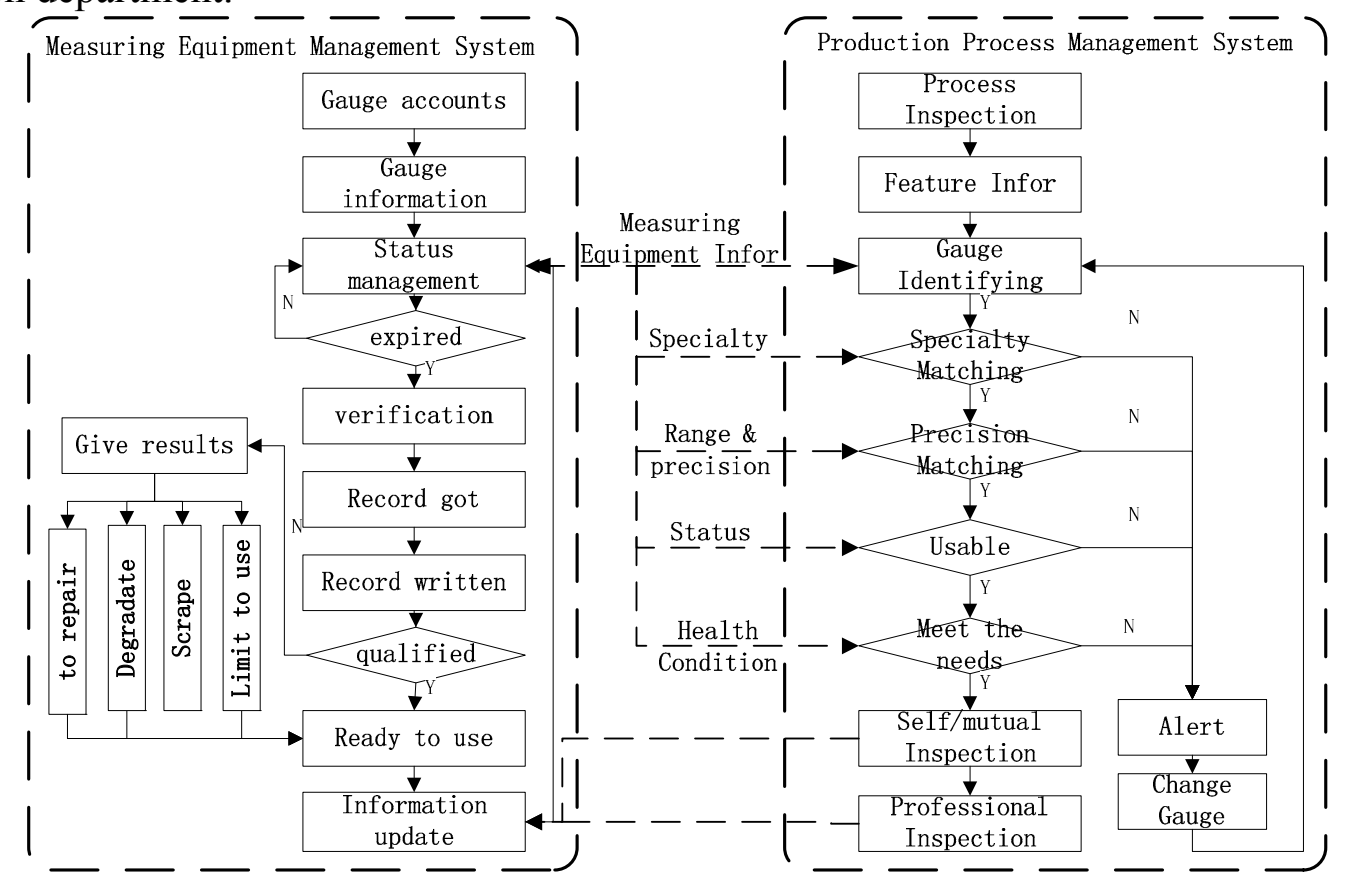

Fig. 2 Assurance of data collection in manufacturing field

In order to get the real-time and accuracy production data the quick identifying technology and some restriction are taken by the measuring equipment management. To get high quality data there are three conditions. Firstly, measuring equipment are healthy and meeting the needs of inspecting. Secondly, the connection between the measuring equipment management system and the production process management system should be built to make preparation of collecting data. Finally, setting restrictions when the inspection process begins to ensure the data is get under correct circumstance. In Fig. 2 there are two systems, the Measuring Equipment Management System controls the gauges' accounts, information, status and periodical verification and their update. To make sure the data has high quality the interaction between the two parts is built to check the measuring equipment to be used. The Information, specialty, range, precision and health condition are matched to the feature information of the product. If there is any factor which is not matched, the System would prompt the operator to change the measuring equipment. The inspection data will be received with the reasonable measuring equipment. As mentioned above, the Assurance of data collection in production field has been realized and the high-quality data can be received and stored for further use.

Measurement System Analysis (MSA).In order to ensure the quality of manufacturing data collected by measuring equipment the capability and performance of measurement system are studied for the most of time of lifecycle for a gauge, which is used to measure the products ${ }^{[5]}$.Measurement System Analysis uses mathematical and experimental method to confirm the measuring variation for both variable measurement system and attribute measurement system, the paper mainly discuss the second system model for the manufacturing data is always continuous and it gives the formulas to get the Repeatability, Reproducibility, GR\&R and \%GR\&R ${ }^{[6]}$. The variation of the measurement system consists of variation from gauges, operators and products, and the properties and structure of the product will not be changed during the process, and the measurement process meets the request of randomness and statistical independence. Fig. 3 shows the flow of MSA by analyzing the properties of the system, and it also give the solution to the 
production control.

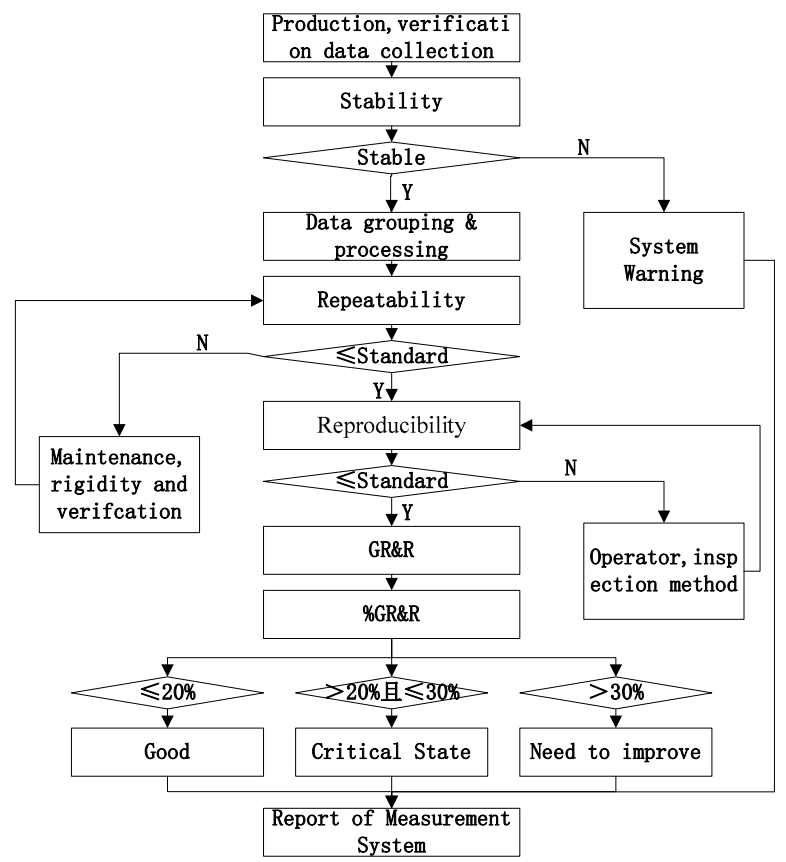

Fig. 3 Measurement System Analysis Flow of the Measuring Equipment Management System for the production process

The measurement system is evaluated by analyzing the variation of gauge, operator and method to get the Repeatability and Reproducibility ${ }^{[7]}$. The same feature of the products from the same batch is inspected by operators, and the system can store these data and relationships. In order to do statistical analysis the data was grouped by the operators and the products for every gauge as Table 1 shows.

Table 1 Data Grouping

\begin{tabular}{|c|c|c|c|c|c|}
\hline Products & $O_{1}$ & $\cdots$ & $O_{i}$ & $\cdots$ & $O_{m}$ \\
\hline \multirow{5}{*}{$P_{1}$} & $x_{111}$ & $\cdots$ & $x_{i 11}$ & $\cdots$ & $x_{m 11}$ \\
\hline & $\cdots$ & $\cdots$ & $\cdots$ & $\cdots$ & \\
\hline & $x_{11 k}$ & $\cdots$ & $x_{i 1 k}$ & $\cdots$ & $x_{m 1 k}$ \\
\hline & $\cdots$ & $\cdots$ & $\cdots$ & $\cdots$ & \\
\hline & $x_{11 p}$ & $\cdots$ & $x_{i 1 p}$ & $\cdots$ & $x_{m 1 p}$ \\
\hline \multirow[t]{3}{*}{$\cdots$} & $\cdots$ & $\cdots$ & $\cdots$ & $\cdots$ & \\
\hline & $x_{1 j 1}$ & $\cdots$ & $x_{i j 1}$ & $\cdots$ & $x_{m j 1}$ \\
\hline & $\cdots$ & $\cdots$ & $\cdots$ & $\cdots$ & \\
\hline \multirow[t]{3}{*}{$P_{j}$} & $x_{1 j k}$ & $\cdots$ & $x_{i j k}$ & $\cdots$ & $x_{m j k}$ \\
\hline & $\cdots$ & $\cdots$ & $\cdots$ & $\cdots$ & \\
\hline & $x_{1 j p}$ & $\cdots$ & $x_{i j p}$ & $\cdots$ & $x_{m j p}$ \\
\hline \multirow[t]{3}{*}{$\cdots$} & $\cdots$ & $\cdots$ & $\cdots$ & $\cdots$ & \\
\hline & $x_{1 n 1}$ & $\cdots$ & $x_{i n 1}$ & $\cdots$ & $x_{m n 1}$ \\
\hline & $\cdots$ & $\cdots$ & $\cdots$ & $\cdots$ & \\
\hline \multirow[t]{3}{*}{$P_{n}$} & $x_{1 n k}$ & $\cdots$ & $x_{i n k}$ & $\cdots$ & $x_{m n k}$ \\
\hline & $\cdots$ & $\cdots$ & $\cdots$ & $\cdots$ & \\
\hline & $x_{1 n p}$ & $\ldots$ & $x_{i n p}$ & $\ldots$ & $x_{m n p}$ \\
\hline
\end{tabular}

-- " $m$ " represents the number of the operators; " $n$ " represents the number of the products; " $p$ " represents the number of the inspection times.

-- $i=1,2, \cdots, m ; j=1,2, \cdots, n ; k=1,2, \cdots, p$;

1) Repeatability Evaluation

Range of subgroup:

$$
R_{i j}=\max \left\{x_{i j 1}, \cdots, x_{i j p}\right\}-\min \left\{x_{i j 1}, \cdots, x_{i j p}\right\}
$$

Average Range:

$$
\bar{R}=\frac{1}{m n} \sum_{i=1}^{m} \sum_{j=1}^{n} R_{i j}
$$


Standard deviation:

Repeatability Variation:

$$
\begin{aligned}
& \quad \sigma_{e}=\frac{\bar{R}}{d_{2} *} \\
& \text {--The value of } d_{2} * \text { can reference the related chart; } \\
& 5.15 \sigma_{e} \\
& \text {--The probability is } 99 \% ;
\end{aligned}
$$

2) Reproducibility Evaluation

The average of the operator $i$ :

$$
\bar{x}_{i}=\frac{1}{n p} \sum_{j=1}^{n} \sum_{k=1}^{p} x_{i j k}
$$

Average Range:

$$
\bar{R}_{0}=R_{0}=\max \left\{\bar{x}_{1}, \cdots, \bar{x}_{m}\right\}-\min \left\{\bar{x}_{1}, \cdots, \bar{x}_{m}\right\}
$$

Reproducibility Variation:

$$
5.15 \frac{\bar{R}_{0}}{d_{2} *}
$$

3) GR\&R Evaluation

Standard deviation of operator:

$$
\sigma_{0}=\frac{\bar{R}_{0}}{d_{2} *}
$$

Standard deviation of measurement system:

$$
\sigma_{m}=\sqrt{\sigma_{e}^{2}+\sigma_{0}^{2}}
$$

Measurement System Variation $(G R \& R)$ :

$$
G R \& R=5.15 \sigma_{m}
$$

4) $\% G R \& R$

$$
\begin{aligned}
\% G R & \& R=\frac{G R \& R}{T V} \times 100 \% \\
& \quad-T V \text { is the total variation of the measurement system and the product. }
\end{aligned}
$$

The formulas (1) (11) is used to analyze the measurement system in the methodology.

\section{Conclusions}

Measuring equipment play a very important part in the manufacturing process, and their application always exits in the whole lifecycle of the products. In this paper, the problems and limitation of the traditional measuring equipment management is proposed, and the methodology of the measuring equipment management for the manufacturing process is showed. The methodology is explained from the perspective of the measuring equipment and the manufacturing process. The management method is detailed with the control process flow and the assurance of the data collection, and the thinking of MSA in the system is introduced to analyze the measuring equipment. Measuring equipment management which considered with manufacturing process has been used in a aviation enterprises and improved the quality of the manufacture, and it has reduced the cost of the production process and the management of the measuring equipment.

In this paper the connection between the measuring equipment management and manufacturing process control has been built, the management can also consider the factors as supplier management and inventory management to improve the quality of manufacturing enterprise.

\section{Acknowledgement}

This work is carried out under the support of National Defense Fundamental Research Foundation, 
China (NO.A2120132007). 


\section{References}

[1] ZHANG Xingwu. Design of Metrology Management System [J]. Nuclear Power Engineering. 2008,29(s1):65-68

[2] LIU Yanbo, CHEN Jianwei. The functional design of metering management information system in the petrochemical plant [J]. Computers and Applied Chemistry. 2010,27(08):1135-1138

[3] TANG Xiaoqing, DUAN Guijiang, DU Fuzhou. Implementing Technologies of Quality Information System in Manufacturing Enterprises [M]. Beijing: National Defense Industry Press, 2009

[4] CHAI Tianyou, Challenges of Optimal Control for Plant-wide Production Processes in Terms of Control and Optimization Theories [J]. Acta Automatica Sinica. 2009,35(6): 641-649

[5] DONG Shuangcai. Measurement System Analysis - theory, method and application[M].Beijing: China Metrology Publishing House, 2006

[6] HadiMoheb-Alizadeh. Capability analysis of the variable measurement system with fuzzy data[J].Applied Mathematical Modelling,2014,38:4559-4573

[7] XI Dayong, CHEN Zhihua. Discussion of measurement system analysis(MSA) in the application of chemical analysis management [J]. Metallurgical Analysis, 2008,28(7): 79-82 